\title{
Ehlers-Danlos syndrome type IV with few extrathoracic findings: a newly recognized point mutation in the COL3A1 gene
}

\author{
A. Watanabe*, Y. Kawabata\#, O. Okada*, N. Tanabe*, H. Kimura*, A. Hatamochi", \\ H. Shinkai ${ }^{\circledR}$, N. Sakai ${ }^{+}$, T. Shimada $^{+}$, K. Hiroshima ${ }^{\S}$, T. Kuriyama*
}

Ehlers-Danlos syndrome type IV with few extrathoracic findings: a newly recognized point mutation in the COL3Al gene. A. Watanabe, Y. Kawabata, O. Okada, $N$. Tanabe, H. Kimura, A. Hatamochi, H. Shinkai, N. Sakai, T. Shimada, K. Hiroshima T. Kuriyama. (C) ERS Journals Ltd 2002.

ABSTRACT: Ehlers-Danlos syndrome type IV (EDS IV) is caused by mutation within the COL3A1 gene, resulting in the disorder of type III procollagen. The diagnosis is confirmed by demonstrating the synthesis of abnormal type III procollagen molecules from cultured dermal fibroblasts or by identifying the mutation in the COL3A1 gene.

The authors report a case of EDS IV caused by a novel point mutation in the COL3A1 gene in a 16-yr-old female. Recurrent haemoptysis and cavitary formation of the lung were evidence of pulmonary involvement. However, extrathoracic manifestations of EDS IV were mostly absent.

To the best of the authors' knowledge, all previously reported Ehlers-Danlos syndrome IV patients with respiratory disease had the characteristic findings or histories of Ehlers-Danlos syndrome IV. In the present case, connective tissue friability was suspected due to tissue laceration observed in the biopsied lung specimen, and the diagnosis was made beginning from this pivotal finding.

Eur Respir J 2002; 19: 195-198.

\begin{abstract}
*Depts of Respirology, "Clinical Biology of Extracellular Matrix, and ${ }^{\S}$ Dept of Basic Pathology, Graduate School of Medicine, Chiba University, Chiba, Japan. " Dept of Laboratory Medicine, Saitama Cardiovascular and Respiratory Centre, Saitama, Japan. ${ }^{+}$Dept of Biochemistry and Molecular Biology, Nippon Medical School, Tokyo, Japan.
\end{abstract}

Correspondence: A. Watanabe, Dept of Respirology, Graduate School of Medicine, Chiba University, 1-8-1 Inohana, Chuou-ku, Chiba City, Chiba 260-8670, Japan

Fax: 81432262176

Keywords: Cavitary formation in the lung, Ehlers-Danlos syndrome type IV, friability of lung tissue, massive haemoptysis

Received: February 272001

Accepted after revision July 32001
Ehlers-Danlos syndrome type IV (EDS IV), the vascular type, is the most severe form of EhlersDanlos syndromes [1]. It is characterized by four diagnostic criteria: easy bruising, thin transparent skin, characteristic facial features, and fatal rupture of the arteries, uterus or intestines, which usually occur at a young age $[2,3]$. The diagnosis is often made following a catastrophic complication or at post mortem examination [4]

Several cases of EDS IV with respiratory manifestations have been reported [5-9]. All of these cases, to the best of the authors' knowledge, were accompanied by such clinical features as characteristic facies, translucent skin, excessive bruising, or a familial history. Described here is the case of a 16-yr-old female with EDS IV for whom the correct diagnosis was difficult to make because of a paucity of extrathoracic findings.

\section{Case report}

A 16-yr-old female, who had been in good health, was referred from another hospital because of recurrent haemoptysis. Her facies and skin were normal. Her parents and brother were also in good health. From 6 months prior to hospitalization, she had experienced recurrent haemoptysis approximately once a month, but there was no relationship to menstruation, and transient numbness of the right side of her body was an accompanying symptom.

Haematological studies showed mild anaemia (haemoglobin concentration was $11.4 \mathrm{~g} \cdot \mathrm{dL}^{-1}$ ), and coagulation studies, including bleeding time, were normal. Chest radiography and computed tomography demonstrated a cavitary lesion in the right lung field (fig. 1), but 1-month later the lesion had spontaneously disappeared (fig. 2). Neurological examinations including brain magnetic resonance imaging and electroencephalography failed to reveal any abnormalities. Pulmonary arteriography was performed to detect any abnormalities in the pulmonary vessels, but the results were negative.

Subsequently, wedge arteriography of the right $\mathrm{A}^{9}$ was performed. Immediately after this procedure, haemothorax and massive haemoptysis developed, which were treated with thoracic drainage and transfusion. The haemothorax caused a residual haematoma and accompanying atelectasis in the right lower lobe. The differential diagnosis included trauma, 


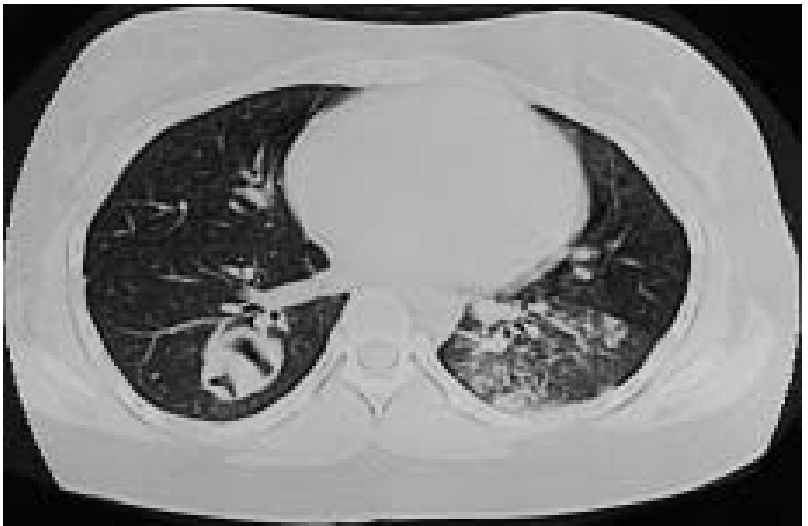

Fig. 1.-Chest computed tomography scan 1 month prior to hospitalization. A cavitary lesion with a thick wall is present in the right lower lobe. In the left lower lobe, an ill-defined opacity with reticular pattern is revealed, which is compatible with pulmonary haemosiderosis.

neoplasm, autoimmune diseases, haematologic disorders, and collagen vascular diseases. Two weeks later, thoracotomy was performed for removal of the haematoma and for open lung biopsy.

The biopsied lung specimen revealed marked diffuse haemorrhage, haemosiderosis, and fibrosis with osseous metaplasia. In the areas of the fibrosis, elastic tissue was lacerated and had already disappeared (fig. 3). There was no evidence of vasculitis or bronchiectasis.

These findings indicated the fragility of the lung tissue. Based on this, a connective tissue disorder was suspected and, after obtaining informed consent, biochemical study of biopsied skin tissue was performed.

Analysis of collagen synthesis using dermal fibroblasts was performed as previously described [10]. The production of normal type III procollagen was remarkably decreased (fig. 4). Genetic analysis was then carried out by examining the coding sequence of messenger ribonucleic acid (mRNA) of cultured

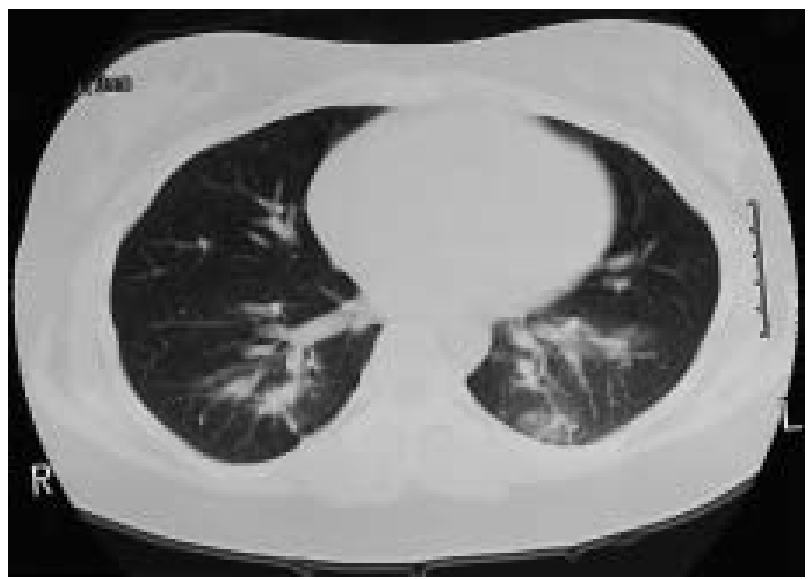

Fig. 2.-Chest computed tomography scan on admission. The cavitary lesion in the right lower lobe is resolved. Small nodular consolidation is present in the area corresponding to the cavitary lesion.

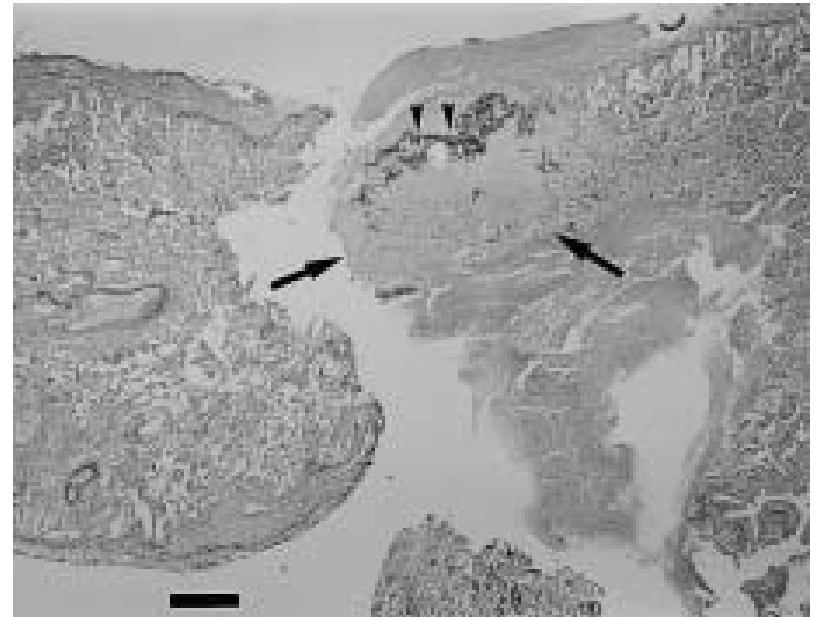

Fig. 3.- Lung biopsy specimen from the patient showing laceration and disappearance of elastic tissue in the subpleural scar (arrow). Destruction of the pleura with secondary organizing pleuritis and secondary pleural elastosis (arrowheads) are also seen. Elastic staining. Internal scale bar $=0.5 \mathrm{~mm}$.

dermal fibroblasts as described [11]. A missense base mutation was revealed in the COL3A1 gene heterozygously, which converted the glycine codon GGT at amino acid position 727 to the codon GAT which codes for aspartate. Based on these findings, a diagnosis of EDS IV was made. Mutation at this location had not been previously reported as a cause of this disorder [12]. Unfortunately, due to the ethical regulations of the authors' institution, confirmation of the genetic status of the patient's parents could not be carried out.

The patient's postoperative course was uneventful. Five months later, she experienced haemothorax again, but she improved by the use of only conservative therapies.

\section{Discussion}

Respiratory system involvement is not common in EDS IV, but when present, pneumothoraces are the most common respiratory complications [6]. Haemoptysis, bulla formation, cavitary pulmonary lesions, and fibrous pseudotumour have also been described [5-9]. This case was distinctive because all previously reported patients had family histories strongly suggestive of EDS IV, physical findings characteristic of the disorder, or episodes of arterial, intestinal or organ rupture.

Neurological complications including epilepsy [13] and temporary hemiparesis [5] developing in patients with EDS IV have also been reported. It has been suggested that some cases of EDS IV have such neurovascular diseases as microangiopathy and various congenital malformations including cortical dysgenesis, which are difficult to detect with the current conventional neuroimaging techniques $[13,14]$. The numbness of the body experienced by the patient studied here might be ascribed to such a disorder.

EDS IV results from mutations in the COL $3 \mathrm{~A} 1$ 


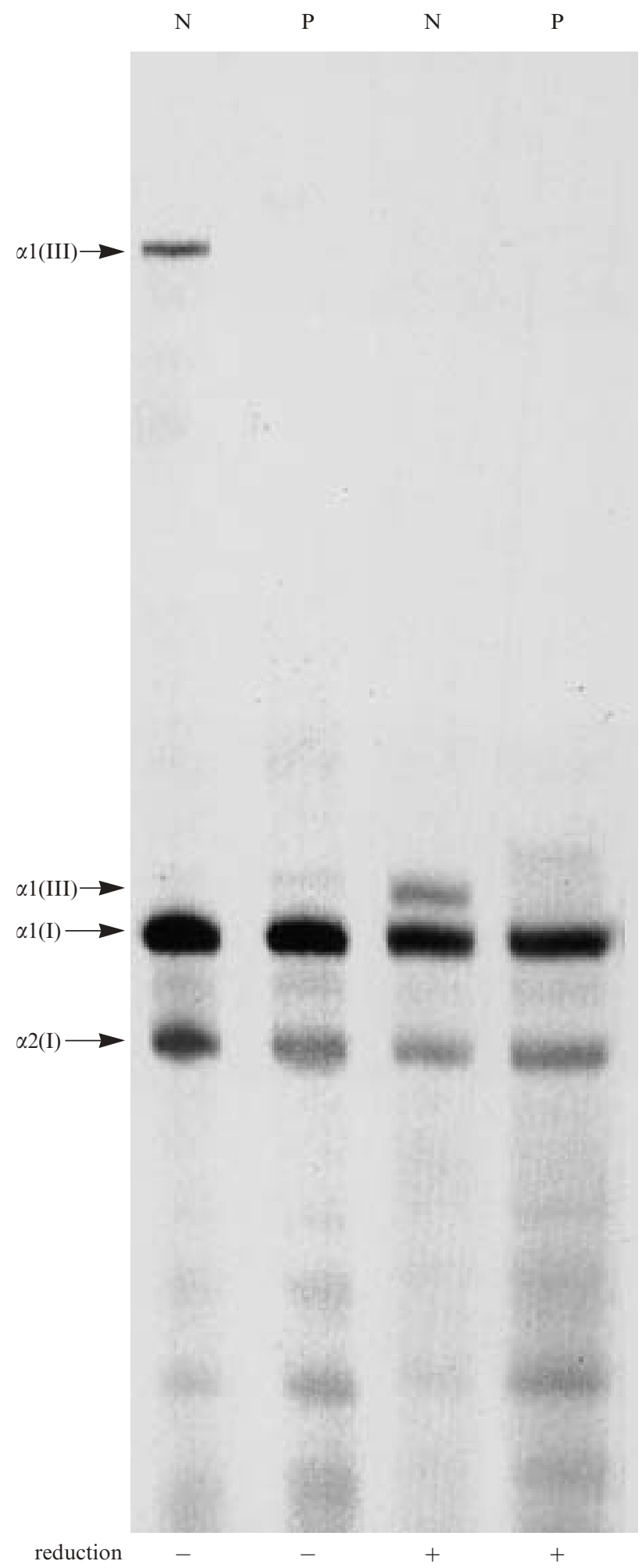

Fig. 4.-Fluorograms of sodium dodecylsulphate (SDS)-polyacrylamide gel electrophoresis of procollagen obtained from dermal fibroblasts of the patient (P) and from those of an age- and sexmatched normal volunteer $(\mathrm{N})$. Both fibroblasts were cultured in appropriate medium. After the collagen samples were treated with $0.1 \%$ pepsin, they were separated by $\mathrm{SDS} / 5 \%$ polyacrylamide gel electrophore in the presence (reduction + ) or absence (reduction -) of 2-mercaptoethanol. Although the patient's fibroblasts produced an equal amount of type I procollagen to normal control fibroblasts, synthesis of type III procollagen from the patient's fibroblasts showed a remarkable decrease. gene which encodes type III procollagen [1, 2], which is a major component of blood vessels, uterus, and intestines $[1,15]$. Fibroblastic cells in the skin of affected individuals have dilated rough endoplasmic reticulum (RER) containing abnormal type III procollagen molecules; in such patients the diameter of collagen fibrils in the skin is small [16]. Type III procollagen is a homotrimer of three identical $\alpha 1$ chains. Therefore, by the heterozygous mutation of COL3A1 gene, wild-type homotrimers comprise only one-eighth of the type III procollagen molecules produced [1].

PEPIN et al. [4] suggested that there was no apparent relationship between the type of complication and the location of mutations. However, it has also been suggested that the nature of the mutation can relate to phenotype [16-23]. Mutations at the very $3^{\prime}$ end of the gene generally produce a severe form of the disease, with arterial rupture and markedly reduced life expectancy, and mutations located in the middle part or at the $5^{\prime}$ end produce more variable phenotypes and milder variants [1, 17, 18]. SMiтH et al. [16] notes that RER dilation and small collagen fibrils in the dermis are more common with mutation near the C-terminal end of the triple helix, whereas variable fibril diameters reflect mutations more toward the N-terminal in the triple helical domain. However, mutation at the very $\mathrm{N}$-terminal end can produce severe vascular involvement [1,4]. EDS IV is almost uniformly fatal because even a single arterial rupture is often lethal.

Many types of mutations in the gene have already been reported [12], but the present mutation (Gly727Asp, the middle part of the protein molecule) has not been previously documented. At a speculative level, if the parents, whose phenotypes are apparently normal, had been available for examination (they were not, for ethical reasons), and no mutations had been found, it could be concluded that the clinical features seen in the present case were due to the uniqueness of the mutation. A complete map of all the mutations and analyses of the clinical features of this disease will be indispensable for eventually proving this hypothesis.

\footnotetext{
Acknowledgements. The authors would like to thank A. Sugito, H. Kawana and A.
} Watanabe.

\section{References}

1. De Paepe A. The Ehlers-Danlos syndrome: a heritable collagen disorder as cause of bleeding. Thromb Haemost 1996; 75: 379-386.

2. Prockop DJ, Kuivaniemi H, Tromp G. Inherited disorders of connective tissue. In: Fauci AS, Braunwald $\mathrm{E}$, Isselbacher $\mathrm{KJ}$, et al. eds. Harrison's principles of internal medicine, 14th Edn. New York, McGrawHill, 1998; pp. 2183-2194

3. Beighton P, De Paepe A, Steinmann B, Tsipouras P, Wenstrup RJ. Ehlers-Danlos syndromes: revised nosology, Villefranche, 1997. Ehlers-Danlos National 
Foundation (USA) and Ehlers-Danlos Support Group (UK). Am J Med Genet 1998; 77: 31-37.

4. Pepin M, Schwarze U, Superti-Furga A, Byers PH. Clinical and genetic features of Ehlers-Danlos syndrome type IV, the vascular type. $N$ Engl J Med 2000; 342: 673-680.

5. Yost BA, Vogelsang JP, Lie JT. Fatal hemoptysis in Ehlers-Danlos syndrome. Old malady with a new curse. Chest 1995; 107: 1465-1467.

6. Dowton SB, Pincott S, Demmer L. Respiratory complications of Ehlers-Danlos syndrome type IV. Clin Genet 1996; 50: 510-514.

7. Corrin B, Simpson CGB, Fisher C. Fibrous pseudotumours and cyst formation in the lungs in EhlersDanlos syndrome. Histopathology 1990; 17: 478-479.

8. Herman TE, McAlister WH. Cavitary pulmonary lesions in type IV Ehlers-Danlos syndrome. Pediatr Radiol 1994; 24: 263-265.

9. Murray RA, Poulton TB, Saltarelli MG, et al. Rare pulmonary manifestation of Ehlers-Danlos syndrome. J Thorac Imaging 1995; 10: 138-141.

10. Hata R, Kurata S, Shinkai H. Existence of malfunctioning pro $\alpha 2(\mathrm{I})$ collagen genes in a patient with a pro $\alpha 2$ (I)-chain-defective variant of Ehlers-Danlos syndrome. Eur J Biochem 1988; 174: 231-237.

11. Tromp G, Wu Y, Prockop DJ, et al. Sequencing of cDNA from 50 unrelated patients reveals that mutations in the triple-helical domain of type III procollagen are an infrequent cause of aortic aneurysms. J Clin Invest 1993; 91: 2539-2545.

12. Dalgleish R. The human collagen mutation database 1998. Nucleic Acids Res 1998; 26: 253-255.

13. Jacome DE. Epilepsy in Ehlers-Danlos syndrome. Epilepsia 1999; 40: 467-473.

14. Jacome DE. Headache in Ehlers-Danlos syndrome. Cephalalgia 1999; 19: 791-796.

15. Clark JG, Kuhn C III, Uitto J. Lung collagen in type IV Ehlers-Danlos syndrome: ultrastructural and biochemical studies. Am Rev Respir Dis 1980; 122: 971-978.

16. Smith LT, Schwarze U, Goldstein J, Byers PH.
Mutations in the COL3A1 gene result in the EhlersDanlos syndrome type IV and alterations in the size and distribution of the major collagen fibrils of the dermis. J Invest Dermatol 1997; 108: 241-247.

17. Mackay K, Raghunath M, Superti-Furga A, Steinmann B, Dalgleish R. Ehlers-Danlos syndrome type IV caused by Gly400Glu, Gly595Cys and Gly1003Asp substitutions in collagen III: clinical features, biochemical screening, and molecular confirmation. Clin Genet 1996; 49: 286-295.

18. Pope FM, Narcisi P, Nicholls AC, Germaine D, Pals G, Richards AJ. COL3A1 mutations cause variable clinical phenotypes including acrogeria and vascular rupture. Br J Dermatol 1996; 135: 163-181.

19. Tromp G, Kuivaniemi H, Stolle C, Pope FM, Prockop DJ. Single base mutation in the type III procollagen gene that converts the codon for glycine 883 to aspartate in a mild variant of Ehlers-Danlos syndrome IV. J Biol Chem 1989; 264: 19313-19317.

20. Gilchrist D, Schwarze U, Shields K, MacLaren L, Bridge PJ, Byers PH. Large kindred with EhlersDanlos syndrome type IV due to a point mutation (G571S) in the COL3A1 gene of type III procollagen: low risk of pregnancy complications and unexpected longevity in some affected relatives. Am J Med Genet 1999; 82: 305-311.

21. Richards AJ, Lloyd JC, Narcisi $\mathrm{P}$, et al. A 27-bp deletion from one allele of the type III collagen gene (COL3A1) in a large family with EhlersDanlos syndrome type IV. Hum Genet 1992; 88: 325-330.

22. McGrory J, Costa T, Cole WG. A novel G499D substitution in the $\alpha 1$ (III) chain of type III collagen produces variable forms of Ehlers-Danlos syndrome type IV. Hum Mutat 1996; 7: 59-60.

23. Nishiyama Y, Nejima J, Watanabe A, et al. EhlersDanlos syndrome type IV with a unique point mutation in COL3A1 and familial phenotype of myocardial infarction without organic coronary stenosis. J Intern Med 2001; 249: 103-108. 